\title{
Lysine221 is the general base residue of the isochorismate synthase from Pseudomonas aeruginosa (PchA) in a reaction that is diffusion limited
}

\author{
Kathleen M. Meneely ${ }^{1}$, Qianyi Luo ${ }^{1}$, Prajnaparamita Dhar ${ }^{2}$, and Audrey L. Lamb ${ }^{1,{ }^{*}}$ \\ ${ }^{1}$ Molecular Biosciences, University of Kansas, Lawrence, Kansas 66045 \\ ${ }^{2}$ Chemical and Petroleum Engineering, University of Kansas, Lawrence, Kansas 66045
}

\begin{abstract}
The isochorismate synthase from Pseudomonas aeruginosa (PchA) catalyzes the conversion of chorismate to isochorismate, which is subsequently converted by a second enzyme (PchB) to salicylate for incorporation into the salicylate-capped siderophore pyochelin. PchA is a member of the MST family of enzymes, which includes the structurally homologous isochorismate synthases from E. coli (EntC and MenF) and salicylate synthases from Yersinia enterocolitica (Irp9) and Mycobacterium tuberculosis (MbtI). The latter enzymes generate isochorismate as an intermediate before generating salicylate and pyruvate. General acid - general base catalysis has been proposed for isochorismate synthesis in all five enzymes, but the residues required for the isomerization are a matter of debate, with both lysine 221 and glutamate 313 proposed as the general base (PchA numbering). This work includes a classical characterization of PchA with steady state kinetic analysis, solvent kinetic isotope effect analysis and by measuring the effect of viscosogens on catalysis. The results suggest that isochorismate production from chorismate by the MST enzymes is the result of general acid - general base catalysis with a lysine as the base and a glutamic acid as the acid, in reverse protonation states. Chemistry is determined to not be rate limiting, favoring the hypothesis of a conformational or binding step as the slow step.
\end{abstract}

\section{Keywords}

Isochorismate synthase; salicylate synthase; siderophore biosynthesis; enzyme kinetics

\section{Introduction}

The menaquinone, $\underline{s}$ iderophore and tryptophan (MST) family of enzymes are structurally homologous, $\mathrm{Mg}^{2+}$-dependent, chorismate-utilizing enzymes that 1) isomerize the ring to form isochorismate for menaquinone and siderophore biosynthesis (Figure 1a), 2) replace the $\mathrm{C} 4-\mathrm{OH}$ with an amine to generate aminodeoxychorismate for folate biosynthesis, or 3 ) remove the $\mathrm{C} 4-\mathrm{OH}$ and add a $\mathrm{C} 2$-amine to produce aminodeoxyisochorismate for tryptophan biosynthesis ${ }^{(1-3)}$. The focus of this study is an enzyme that catalyzes the first reaction, PchA, the isochorismate synthase from Pseudomonas aeruginosa. The isochorismate that is

\footnotetext{
(C) 2013 Elsevier Inc. All rights reserved.

*corresponding author; phone: (785)864-5075; fax: (785)864-5294; lamb@ku.edu.
}

Publisher's Disclaimer: This is a PDF file of an unedited manuscript that has been accepted for publication. As a service to our customers we are providing this early version of the manuscript. The manuscript will undergo copyediting, typesetting, and review of the resulting proof before it is published in its final citable form. Please note that during the production process errors may be discovered which could affect the content, and all legal disclaimers that apply to the journal pertain. 
produced by PchA is subsequently converted to salicylate and pyruvate by the isochorismate-pyruvate lyase PchB (Figure 1b), and the salicylate is incorporated into the salicylate-capped siderophore pyochelin $(4,5)$. Other isochorismate synthases have been characterized, including EntC ${ }^{6,7)}$, which is involved in the formation of the dihydroxybenzoate-capped siderophore enterobactin, and $\mathrm{MenF}^{(2)}$, which is in the pathway for formation of the electron-carrier menaquinone (Vitamin K), both from Escherichia coli. Irp9 and MbtI are salicylate synthases (Figure 1c) involved in salicylate-capped siderophore biosynthesis, yersiniabactin of Yersinia enterocolitica ${ }^{(8-10)}$ and mycobactin of Mycobacterium tuberculosis $(3,11)$, respectively. These latter two enzymes generate an isochorismate intermediate using a general acid - general base mechanism hypothesized to be common to all five, but also have isochorismate-pyruvate lyase activity to form salicylate $^{(3,9,11)}$. The structures of $\operatorname{MenF}^{(2)}, \operatorname{EntC}^{(6,7)}, \operatorname{Irp9}{ }^{(8)}$ and $\operatorname{MbtI}{ }^{(3,11,12)}$ have been determined and the active sites are shown to be architecturally similar and built from highly conserved amino acids. The structure of PchA remains elusive but is hypothesized to be homologous based on sequence conservation, especially in the active site amino acids (13).

The formation of isochorismate by both the isochorismate and salicylate synthases has been hypothesized to be catalyzed by a general acid - general base mechanism shown in Figure 1a $(2,3,8,14,15)$. The proposal $(2,3)$ was that a lysine residue $(221$ in PchA) served as the general base, accepting a proton from water, thereby activating the water molecule for nucleophilic attack at the 2 position of the substrate ring. The hydroxyl at the 4 position accepts a proton from a glutamic acid general acid (269 in PchA) thereby making it a good leaving group. The lysine general base residue was chosen based on the crystal structures of MST enzymes which showed this residue to be in proximity to a water molecule in the appropriate position for activation and catalysis $(2,3,9,15)$. This family-wide hypothesis (1) has found support from mutational analyses in MenF ${ }^{(2)}$, Irp9 ${ }^{(8)}$ and MbtI ${ }^{(3)}$ that showed a loss of isochorismate synthase activity in variants in which the general base lysine was replaced with alanine or the general acid glutamic acid was replaced with alanine or glutamine. Recent work by Ziebart and Toney ${ }^{(15)}$ on EntC and MbtI puts this hypothesis in question, as they found that lysine to glutamine variants retained some activity. A second general acid - general base proposal based on a computational model of MbtI ${ }^{(14)}$ suggests that the general base is instead a glutamic acid (313 in PchA): the lysine was calculated to have a $\mathrm{p} K_{\mathrm{a}}$ of 10.9 , and thus was reasoned to be protonated in the active site and unsuitable as a general base, whereas the glutamic acid was calculated to have a $\mathrm{p} K_{\mathrm{a}}$ value of 6.55 and thus be more likely to accept a proton at physiological $\mathrm{pH}$. It should be noted that these calculations were conducted using a model based on a structure lacking the catalytically required magnesium ion. Other structures from the MST family members (Irp9, MenF) show that the glutamic acid proposed to serve as a base (equivalent to E313 in PchA) is involved in metal ion chelation. To directly test the hypotheses of general acid - general base catalysis in this family, we document here the first solvent isotope effects experiments for any member of this family, specifically PchA. These data, in conjunction with viscosogen data, are most consistent with the original model of a lysine general base and a glutamic acid general acid, with these two residues in reverse protonation states.

\section{Materials and Methods}

\section{Preparation of overexpression plasmids}

The pch $A$ gene was amplified from $P$. aeruginosa PA01 genomic DNA by polymerase chain reaction by use of Master Mix (Eppendorf). The forward primer (Table 1) includes an NdeI site (underlined), whereas the reverse primer contains a HindIII site (underlined). The amplified 1431 base pair fragment was digested with NdeI and HindIII and ligated into the pET28b plasmid (Novagen) digested with the same enzymes. The resulting plasmid contains 
the $p c h A$ gene with an $\mathrm{N}$-terminal histidine tag. The PchA variants were produced using QuikChange (Agilent) with wildtype $p c h A$ plasmid as the template with primers as shown in Table 1. Each variant plasmid only contained the desired mutation.

\section{Protein overexpression and purification}

BL21 (DE3) pLysS E. coli containing the PchA expression plasmid were grown in terrific broth medium supplemented with $0.4 \%$ (v/v) glycerol and containing $50 \mu \mathrm{g} / \mathrm{mL}$ kanamycin at $37^{\circ} \mathrm{C}$ with shaking $(225 \mathrm{rpm})$ until an $\mathrm{OD}_{600}$ of $\sim 1.0$ was reached. The culture was induced with $0.2 \mathrm{mM}$ isopropyl $\beta$-D-thiogalactopyranoside and temperature was reduced to $25^{\circ} \mathrm{C}$. The cells were harvested by centrifugation $\left(6,000 \mathrm{xg}, 10 \mathrm{~min}, 4^{\circ} \mathrm{C}\right)$ after $20-22 \mathrm{~h}$. The cell pellet was resuspended in $15 \mathrm{~mL}$ of $25 \mathrm{mM}$ Tris- $\mathrm{HCl} \mathrm{pH} 8,500 \mathrm{mM} \mathrm{KCl}, 5 \mathrm{mM}$ imidazole, $20 \%$ glycerol (buffer A) per liter of culture. Cells were disrupted by use of a French pressure cell $(35,000 \mathrm{psi})$, and cellular debris was removed by centrifugation $\left(12,000 \mathrm{xg}, 45 \mathrm{~min}, 4^{\circ} \mathrm{C}\right)$. The supernatant was applied to a chelating Sepharose fast-flow column (Amersham Biosciences) charged with nickel chloride and pre-equilibrated in buffer A. PchA protein eluted at $300 \mathrm{mM}$ imidazole in a step gradient of $30 \mathrm{mM}, 45 \mathrm{mM}, 150 \mathrm{mM}$ and $300 \mathrm{mM}$ imidazole in buffer A. The pooled fractions were applied to a Superdex 200 size-exclusion column (Amersham Biosciences) equilibrated with $50 \mathrm{mM}$ Tris-HCl pH 8, 50 $\mathrm{mM} \mathrm{KCl}, 50 \mathrm{mM}$ sodium citrate, $10 \%$ glycerol. The fractions containing PchA were pooled and concentrated by use of an Amicon stirred cell with a YM-30 membrane to $5-25 \mathrm{mg} / \mathrm{mL}$ as determined by the Bradford assay and stored at $-80^{\circ} \mathrm{C}$. PchA variants were overexpressed and purified in the same manner as wildtype PchA. PchB for use in the coupled assay with PchA was purified as previously described ${ }^{(16)}$.

\section{Preparation of isochorismate and chorismate}

Isochorismate was isolated from Klebsiella pneumoniae 62-1 harboring the ent $C$ plasmid pKS3-02 ${ }^{(17)}$ with only minor changes, as described previously ${ }^{(18)}$. Chorismate was isolated from $K$. pneumoniae $62-1$ as previously described ${ }^{(19)}$ with few modifications. Briefly, growth medium ( $1 \mathrm{~L}$ ) was inoculated with $10 \mathrm{ml}$ of $K$. pneumoniae 62-1 overnight culture (grown at $30{ }^{\circ} \mathrm{C}$ with shaking at $250 \mathrm{rpm}$ ) and incubated at $30^{\circ} \mathrm{C}$ with shaking $(250 \mathrm{rpm}$ ) until $\mathrm{OD}_{625}$ of $\sim 2.0$. Growth medium contained per liter: $0.2 \mathrm{~g} \mathrm{MgSO}_{4} \cdot 7 \mathrm{H}_{2} \mathrm{O}, 2 \mathrm{~g}$ citric acid monohydrate, $10 \mathrm{~g}$ anhydrous $\mathrm{K}_{2} \mathrm{HPO}_{4}, 3.5 \mathrm{~g} \mathrm{NaNH} 4 \mathrm{PO}_{4} \cdot 4 \mathrm{H}_{2} \mathrm{O}, 2 \mathrm{~g}$ yeast extract, $2 \mathrm{~g}$ casamino acids, and $41 \mathrm{mg}$ DL-tryptophan. Glucose (10 $\mathrm{ml}$ of $16 \%(\mathrm{w} / \mathrm{v}))$ was added after autoclaving. The cells were harvested by centrifugation $\left(6,000 \mathrm{x} g, 10 \mathrm{~min}, 4^{\circ} \mathrm{C}\right)$ and resuspended in $1 \mathrm{~L}$ accumulation medium. Accumulation medium contained per liter: $12.8 \mathrm{~g}$ $\mathrm{Na}_{2} \mathrm{HPO}_{4}, 1.36 \mathrm{~g} \mathrm{KH}_{2} \mathrm{PO}_{4}, 18 \mathrm{~g}$ glucose, $2.7 \mathrm{~g} \mathrm{NH}_{4} \mathrm{Cl}, 2 \mathrm{ml}$ of a $50 \mathrm{mM}$ solution $\mathrm{MgCl}_{2}$, $20.5 \mathrm{mg}$ DL-tryptophan, $1 \mathrm{ml}$ of a $100 \mathrm{mM}$ solution L-phenylalanine, and $1 \mathrm{ml}$ of a $10 \mathrm{mM}$ solution $\left(\mathrm{NH}_{4}\right)_{2} \mathrm{Fe}\left(\mathrm{SO}_{4}\right)_{2}$. Accumulation medium was prepared fresh and not autoclaved. After incubated at $30^{\circ} \mathrm{C}$ with shaking $(250 \mathrm{rpm})$ for 16 to $18 \mathrm{~h}$, the culture was centrifuged $\left(6,000 \mathrm{x} g, 20 \mathrm{~min}, 4^{\circ} \mathrm{C}\right)$, the supernatant collected, and the $\mathrm{pH}$ adjusted to 8.5 by slowly adding $10 \mathrm{M} \mathrm{NaOH}$ or $\mathrm{NaOH}$ pellets on ice to prevent the sample from heating. The accumulation culture supernatant $(5 \mathrm{~L})$ was mixed with approximately $150 \mathrm{ml}$ of Dower-Cl $1 \times 8$ resin (Sigma) and stirred for $30 \mathrm{~min}$ at $4{ }^{\circ} \mathrm{C}$. The resin with chorismate bound was washed with $300 \mathrm{ml}$ of $\mathrm{H}_{2} \mathrm{O}$ three times and packed into an XK 50/20 column (GE Healthcare). The chorismate was eluted with three column volumes of $2 \mathrm{M} \mathrm{NH}_{4} \mathrm{Cl} \mathrm{pH} 8.5$ at $3 \mathrm{ml} / \mathrm{min}$ after equilibration with 1 column volume of $\mathrm{H}_{2} \mathrm{O}$. Fractions $(10 \mathrm{ml})$ were collected and any fraction with the $\mathrm{OD}_{275}$ over 0.3 ( 1 to 100 dilution) was pooled and the $\mathrm{pH}$ adjusted to 1.5 with $10 \mathrm{M} \mathrm{HCl}$. The chorismate was extracted with ether and crystallized as previously described ${ }^{(19)}$. After a recrystallization process, the final yield of chorismate was $200 \mathrm{mg}$ per liter of culture and $>90 \%$ pure as evaluated by HPLC and ${ }^{1} \mathrm{H}-\mathrm{NMR}$ analyses. 


\section{Isochorismate synthase activity assays}

The standard assay buffer contained $50 \mathrm{mM}$ Tris- $\mathrm{HCl} \mathrm{pH} 7.5,10 \mathrm{mM} \mathrm{MgCl}_{2}, 10 \%$ glycerol. Enzyme was added and incubated with a 20 -fold excess of PchB and the reaction was initiated by the addition of chorismate $(0-300 \mu \mathrm{M})$. Initial velocities were determined at 25 ${ }^{\circ} \mathrm{C}$ by measuring the accumulation of salicylate by fluorescence with an excitation wavelength of $310 \mathrm{~nm}$ and an emission wavelength of $430 \mathrm{~nm}$ using a Cary Eclipse fluorescence spectrophotometer (Varian) and an electrothermal single cell holder for temperature control $\left(0.1^{\circ} \mathrm{C}\right)$. For the wildtype PchA assays, the enzyme concentration was $0.1 \mu \mathrm{M}$, and for the PchA variants, the enzyme concentration was $10 \mu \mathrm{M}$. For the PchA solvent isotope effect assays, the reaction buffer contained $50 \mathrm{mM}$ formate, $50 \mathrm{mM}$ MES, $100 \mathrm{mM}$ Tris- $\mathrm{HCl}, 10 \mathrm{mM} \mathrm{MgCl} 2,10 \%$ glycerol at pH 6-9 with an enzyme concentration of $0.1 \mu \mathrm{M}$.

\section{Isochorismate-pyruvate lyase activity assays}

Isochorismate-pyruvate lyase assays were performed using a TgK Scientific Stopped-Flow device operated at $25{ }^{\circ} \mathrm{C}$, with a xenon lamp with the monochromator set at $310 \mathrm{~nm}$, and a $360 \mathrm{~nm}$ cutoff filter. Equal volumes of enzyme and substrate were injected into a $22 \mu \mathrm{l}$ cell and initial velocity of salicylate production was collected for 30 seconds during which the reaction was linear. Pre-injection enzyme and substrate concentrations were twice the final enzyme/substrate concentration in the cell (post mixing). Unless otherwise specified, all concentrations reported are final post-mixing concentrations. Both the isochorismate substrate and enzyme were prepared in the same standard reaction buffer to prevent buffer dilution effects post mix. Isochorismate was prepared to a desired post-mix final concentration of $0-400 \mu \mathrm{M}$ and enzyme concentrations used for the assays with wildtype PchA and PchA variants were $10 \mu \mathrm{M}$.

\section{Salicylate synthase activity assays}

Salicylate production with wildtype PchA and PchA variants was measured using $10 \mu \mathrm{M}$ enzyme and the reaction was initiated with $1 \mathrm{mM}$ chorismate. The different enzymes or variants were added individually to the standard reaction buffer. Initial velocities were determined at $25^{\circ} \mathrm{C}$ by measuring the accumulation of salicylate by fluorescence with an excitation wavelength of $310 \mathrm{~nm}$ and an emission wavelength of $430 \mathrm{~nm}$ using a Cary Eclipse fluorescence spectrometer (Varian) with temperature controller.

\section{Chorismate mutase activity assays}

Initial velocities were measured in $50 \mathrm{mM}$ Tris- $\mathrm{HCl} \mathrm{pH} 7.5,10 \mathrm{mM} \mathrm{MgCl}_{2}, 10 \%$ glycerol or with $50 \mathrm{mM}$ Tris- $\mathrm{HCl} \mathrm{pH} 7.5,1 \mathrm{mM}$ EDTA, $10 \%$ glycerol at $25^{\circ} \mathrm{C}$ with $25 \mu \mathrm{M}$ enzyme according to the method optimized by Gaille et. al ${ }^{(4)}$. The reaction was initiated by the addition of chorismate, varied in concentration from 0 to $10 \mathrm{mM}$. At the various time points, $40 \mu \mathrm{l}$ of reaction mixture was removed and the reaction was terminated with $20 \mu \mathrm{l} 1 \mathrm{M} \mathrm{HCl}$. The reaction product, prephenate, was converted to phenylpyruvate upon incubation at 37 ${ }^{\circ} \mathrm{C}$ for $20 \mathrm{~min}$. The phenylpyruvate concentration was determined at $320 \mathrm{~nm}$ under basic conditions by the addition of $140 \mu \mathrm{l}$ of $2.5 \mathrm{M} \mathrm{NaOH}\left(\epsilon_{320}=17,500 \mathrm{M}^{-1} \mathrm{~cm}^{-1}\right)$ using a Cary 50 Bio spectrophotometer (Varian) with an electrothermal single cell holder for temperature control $\left( \pm 0.1^{\circ} \mathrm{C}\right)$.

\section{Viscosity measurements}

Solvent viscosities in $50 \mathrm{mM}$ Tris $\mathrm{pH} 7.5,10 \mathrm{mM} \mathrm{MgCl} 2$ were determined using a digital cone-and-plate viscometer (DVII+ Pro, CP-40 0.8 cone, Brookfield Engineering, Middleboro, MA). The temperature was controlled at $25^{\circ} \mathrm{C}$. A sample of $0.6 \mathrm{ml}$ was used for all measurements at a final shear rate of $60 \mathrm{rpm}$, while an initial sweep of shear rates was 
done to ensure that the measurements are in the linear regime. The torque was always at greater than $10 \%$ to ensure accuracy of the data. Viscosities are calculated by in-built software using well-established relations between the applied shear stress, shear rate and viscosity. The reported viscosities are averages for 4-5 measurements for each solution: $\eta_{\text {avg }}$ $=1.32,1.88$, and $2.75 \mathrm{cP}$ for 10,20 , and $30 \%(\mathrm{v} / \mathrm{v})$ glycerol solutions, respectively; $\eta_{\text {avg }}=$ $1.76,2.44$ and $4.27 \mathrm{cP}$ for 20,30 , and $40 \%$ (w/v) sucrose solutions; $\eta_{\text {avg }}=1.40,1.84$, and $2.59 \mathrm{cP}$ for 10,20 , and $30 \%(\mathrm{w} / \mathrm{v})$ sorbitol solutions; $\eta_{\mathrm{avg}}=1.33$ and $1.95 \mathrm{cP}$ for 10 and $20 \%$ $(\mathrm{v} / \mathrm{v})$ poly(ethylene glycol) 200 solutions; $\eta_{\mathrm{avg}}=1.52,1.96$, and $5.33 \mathrm{cP}$ for $1.7,3.3$, and $6.7 \%(\mathrm{w} / \mathrm{v})$ poly(ethylene glycol) 8000 solutions. Kinetic parameters in different viscosogen solutions were determined with the isochorismate synthase activity assay using a $\operatorname{TgK}$ SF-61DX2 stopped-flow apparatus with a final enzyme concentration of $0.1 \mu \mathrm{M}$ for wildtype PchA and $25 \mu \mathrm{M}$ for PchB at $25^{\circ} \mathrm{C}$. Final chorismate concentrations ranged from 0 $-10 \mu \mathrm{M}$. The microviscogen data used to determine the slope of the line include the sucrose, PEG200, and sorbitol values. The glycerol data were omitted from the curve fit because they are classically known to be non-linear ${ }^{(20)}$, as can be seen in these data also. The PEG8000 data were used to determine the slope of the line for the macroviscogens.

\section{Data analysis}

Kinetic parameters were determined using the Michaelis-Menten equation with the nonlinear regression function of KaleidaGraph (Synergy Software). Henderson and Dixon plots were used at sub-saturating concentrations of substrate ${ }^{(21)}$. $\mathrm{pH}$ plots were fit to the equation

$$
\log y=\log \left[\frac{c}{1+\left(\frac{H}{K_{a 1}}\right)+\left(\frac{K_{a 2}}{H}\right)}\right]
$$

where $y$ is the observed kinetic parameter $\left(k_{\mathrm{cat}}\right.$ or $\left.k_{\mathrm{cat}} / K_{\mathrm{m}}\right), c$ is $\mathrm{pH}$ independent value of $y$, and $H$ is the proton concentration. Buffers in $\mathrm{D}_{2} \mathrm{O}$ were corrected for isotope effects on the glass electrode function using the equation

$$
p D_{n}=(\text { meter } \quad \text { reading })+(\Delta p H)_{n}
$$

where

$$
(\Delta p H)_{n}=0.076 \quad n^{2}+0.3314 \quad n
$$

and where $n$ is the fraction of $\mathrm{D}_{2} \mathrm{O}{ }^{(22)}$.

\section{Results}

\section{PchA purification and isochorismate synthase wildtype kinetic parameters}

PchA was previously shown to be an isochorismate synthase enzyme that converts chorismate into isochorismate and was reported to be expressed only in conjunction with expression of $\mathrm{PchB}{ }^{(5,23)}$. In our hands, PchA containing an $\mathrm{N}$-terminal His tag allowed for $\sim 90 \mathrm{mg}$ of PchA to be purified per liter of culture without coexpression of PchB, resulting in $>98 \%$ purity after two chromatographic steps. The isochorismate synthase activity of PchA requires $\mathrm{Mg}^{2+(24)}$ and exhibits Michaelis-Menten kinetics. Isochorismate synthase kinetic parameters were determined for wildtype and variant PchA using a coupled assay in which isochorismate produced by PchA is converted to the fluorescent salicylate by the isochorismate-pyruvate lyase, $\mathrm{PchB}{ }^{(5)}$. PchB is used in 20 -fold molar excess such that any isochorismate produced is immediately converted into salicylate. The previous 
characterization of PchA upon co-purification with PchB gave comparable kinetic values, after consideration for differences in temperature and assay buffer ${ }^{(5)}$.

\section{Purification of variants of PchA}

The variants of PchA were purified in the same manner as the wild type enzyme. The yield per liter of culture for the variant enzyme was $90-270 \mathrm{mg}$, with comparable purity.

\section{Isochorismate-pyruvate lyase kinetic parameters}

Isochorismate-pyruvate lyase activity (Figure 1b) had not been previously documented for PchA. Using an assay measuring the production of the fluorescent salicylate product (excitation $310 \mathrm{~nm}$ and emission $>360 \mathrm{~nm}$ ) from an isochorismate substrate, kinetic parameters could be determined: $k_{\text {cat }}=0.00070 \pm 0.00001 \mathrm{~s}^{-1}, K_{\mathrm{m}}=42 \pm 2 \mu \mathrm{M} ; k_{\text {cat }} / K_{\mathrm{m}}=$ $17 \pm 1 \mathrm{M}^{-1} \mathrm{~s}^{-1}$.

\section{Chorismate mutase activity}

Chorismate mutase activity (Figure 1d) has previously been detected for the salicylate synthase enzyme MbtI but only in the absence of magnesium ${ }^{(3)}$. A more recent study did not detect any chorismate mutase activity for MbtI or EntC ${ }^{(15)}$. We did not detect chorismate mutase activity for PchA or any of the variants in the presence or absence of magnesium.

\section{E269A-PchA (loss of general acid) and K221A- or E313(A/Q)-PchA (loss of general base) variants}

Generation of a variant of the proposed general acid in PchA, E269A, yielded enzyme with no detectable isochorismate synthase activity (Table 2) as was also demonstrated with the isochorismate synthase homolog $\mathrm{MenF}^{(2)}$ and the salicylate synthase homologs $\operatorname{Irp} 9{ }^{(8)}$ and MbtI ${ }^{(3)}$. Mutation of the two proposed general base residues of PchA to alanine, K221A and E313A, yielded variants with detectable isochorismate synthase activity that was 4 orders of magnitude less than the wildtype enzyme. This is in contrast to the similar lysine to alanine variants of $\mathrm{MenF}^{(2)}$ or MbtI ${ }^{(3)}$, for which activity was not detected. A mutation of lysine to glutamine at the same position in EntC and MbtI showed 5-8\% of the activity of wildtype ${ }^{(15)}$, higher than seen for the alanine variant. The comparable variant in PchA, E313Q, showed a decrease in activity of three orders of magnitude. The mutational analyses are not definitive for either model (a lysine or glutamic acid general base). Indeed, they cannot be, since a loss of activity upon generation of a variant may be the result of a small or large perturbation in structural integrity, due to changes in binding affinity, or due to the loss or gain in the proposed catalytic property.

\section{PchA pH rate profile and solvent kinetic isotope effects}

Isochorismate synthase kinetic parameters were determined for wildtype $\mathrm{PchA}$ at $\mathrm{pH}$ values 6 to 9 in $\mathrm{H}_{2} \mathrm{O}$. The coupled assay described above was again used. PchB has been shown previously to have constant activity throughout the $\mathrm{pH}$ range tested ${ }^{(25)}$. The secondary structure of PchA was monitored at the $\mathrm{pH}$ extremes and found to be intact by circular dichroism, but the protein was not stable at $\mathrm{pH}$ values below 6 or above 9 (data not shown). A plot of the $\log k_{\text {cat }} / K_{\mathrm{m}}$ versus $\mathrm{pH}$ for PchA shows a classic bell curve with optimal activity at $\mathrm{pH} 7.5$. The curve had apparent slopes of +1 and -1 on the respective acidic and basic sides of the profile. Fitting of the data produced $\mathrm{p} K_{\mathrm{a}}$ values of 5.43 and 9.92 (Figure 2a circles and Table 3 ). The plot of $\log k_{\mathrm{cat}}$ versus $\mathrm{pH}$ was also defined by a bell-shaved curves with slopes of +1 and -1 , which upon fitting gave $\mathrm{p} K_{\mathrm{a}}$ values of 5.61 and 9.74 (Figure $2 \mathrm{~b}$ circles and Table 3). Similarly, plots of $\log k_{\mathrm{cat}} / K_{\mathrm{m}}$ or $\log k_{\mathrm{cat}}$ versus pD (Figure $2 \mathrm{a}$ and $2 \mathrm{~b}$ squares) showed a shift in the $\mathrm{p} K_{\mathrm{a}}$ values $(\Delta \mathrm{p} K)$ of $0.3-0.6$ as expected (Table 3$)^{(26)}$. We 
note that the $\mathrm{p} K_{\mathrm{a}}$ values are outside the $\mathrm{pH}$ range tested. Nevertheless, the values are consistent in all of the experiments, and the small differences in the values do not change the outcome of the calculations found in the discussion section.

The determined $\mathrm{p} K_{\mathrm{a}}$ values are consistent with glutamic acid and lysine residues in a general acid - general base mechanism with reverse protonation states. In other words, K221 is the general base yet the $\mathrm{p} K_{\mathrm{a}}$ is observed for this residue at the basic side of the $\mathrm{pH}$ profile, and E269 is the general acid with the $\mathrm{p} K_{\mathrm{a}}$ for this residue observed at the acidic side of the profile. This is not uncommon in enzymes where the protonation state is different in the forward and reverse reactions (27-30). The isochorismate synthases, in particular $\operatorname{EntC}^{(31,32)}$, MenF ${ }^{(31)}$, and PchA ${ }^{(5)}$, have been documented to be efficient at both the forward and reverse reactions.

In $95 \% \mathrm{D}_{2} \mathrm{O}$ over the same $\mathrm{pH}$ range, a negligible $\mathrm{D}_{\left(k_{\text {cat }} / K_{\mathrm{m}}\right)}$ solvent kinetic isotope effect $(1.09 \pm 0.06)$ was determined (Figure $2 \mathrm{a}$ diamonds and Table 3$)$. The values of $k_{\text {cat }} / K_{\mathrm{m}}$ for PchA in $\mathrm{H}_{2} \mathrm{O}, 50 \% \mathrm{D}_{2} \mathrm{O}$ and $\mathrm{D}_{2} \mathrm{O}$ were within $10 \%$ of each other, suggesting that the proton transfer steps are not rate-limiting. A small, normal ${ }^{\mathrm{D}}\left(k_{\text {cat }}\right)$ solvent kinetic isotope effect of $1.25 \pm 0.3$ was determined (Figure $2 \mathrm{~b}$ diamonds and Table 3 ). This value is more than likely inflated relative to the $k_{\text {cat }} / K_{\mathrm{m}}$ value as a result of the viscosity difference between $\mathrm{H}_{2} \mathrm{O}$ and $\mathrm{D}_{2} \mathrm{O}^{(33)}$ and the large viscosity effect for this enzyme described below. A general acid general base mechanism with a proton transfer occurring in the rate-determining step would suggest that significant solvent isotope effects would be observed with kinetic parameters reduced by up to 4-5 fold with deuterium oxide as solvent. Thus, solvent isotope effects would be expected if proton transfer from the nucleophile water at C2 to K221 or E313 and/ or proton transfer to the departing hydroxyl at C4 from E269 is rate-limiting in the reaction. If these proton transfers occur in relatively fast steps, then the solvent isotope effects would be small, which seems more likely from these data.

\section{Viscosogen data}

Since the chemistry step (general acid - general base) is shown above to not be rate-limiting, a viscosogen study was initiated to determine if the enzyme is limited by diffusive effects if substrate binding or product release are rate-limiting (Figure 3). We should note that these data are also collected using a coupled assay with PchB in 200-fold excess (in contrast to 20 -fold excess in the previous experiments) to convert the product isochorismate of the PchA reaction into the fluorescently detectable salicylate. The dependence of $k_{\text {cat }}$ with respect to viscosogens indicates that the reaction is diffusion controlled, or that the substrate on rate or product off rate has been slowed. Previous documentation suggests that data of this nature is only reliable for slopes between the ranges of 0.2 and 0.8 , and that slopes close to 0 or 1 are error prone and insufficient for the determination rate constants ${ }^{(34)}$.

Nevertheless, the data qualitatively indicate that in the presence of microviscogens (sucrose, glycerol, sorbitol, PEG200), the reaction is diffusion controlled (a slope of $\sim 1$ ) ${ }^{(35,36)}$. In the presence of macroviscogens (PEG8000), the slope is $\sim 0$, suggesting that the effects on substrate binding or product release are not due to major protein conformational changes.

\section{Discussion}

Four possible mechanisms for this class of enzymes were originally proposed based on data for EntC, the isochorismate synthase from E. coli ${ }^{(37)}$. In all four, it was assumed that the magnesium ion chelates the 4-hydroxyl-group of substrate to make it a good leaving group or is part of a magnesium bound transition state ${ }^{(37)}$. However, all of the structures of the MST enzymes in which ligands are bound (substrate or product) have shown that the magnesium is chelated by the carboxylate attached at the 1-position of the ring ${ }^{(7,8,38)}$. A second proposal suggested general acid-general base chemistry with nucleophilic attack at 
$\mathrm{C} 2$ and elimination of the $\mathrm{C} 4-\mathrm{OH}^{(1)}$, assisted by the development of a partial positive charge to increase the electrophilicity at $\mathrm{C} 2$ due to chelation of the $\mathrm{C} 1$-carboxylic acid to the magnesium ion (Figure 1a) ${ }^{(3)}$. A lysine general base residue and a glutamic acid general acid were chosen based on the crystal structures of MST enzymes based on proximity and positioning for activation and catalysis (Figure 4 , residues in yellow) $(2,3,9,15)$. A third mechanistic hypothesis has a basis in the computational determination of $\mathrm{p} K_{\mathrm{a}}$ values of residues in the active site of MbtI, and proposes two glutamic acid residues serving in the roles of general base and general acid (Figure 4, orange glutamic acid is general base) ${ }^{(14)}$. Mutagenic analyses previously conducted $(2,3,8,9,15)$ and herein show that changes at the hypothesized sites do reduce or abrogate catalytic activity. However, a mutationally induced loss of activity suggests only some catalytic role for the residue of interest, among which may be acid/base function or a critical hydrogen bonding, electrostatic, or steric interaction.

To test the hypothesis of general acid - general base chemistry more explicitly, pH-rate profiles and solvent isotope effect experiments of the isochorismate synthase PchA were performed (Figure 2). The $\mathrm{pH}$ rate profiles indicate that one catalytic residue must be deprotonated for maximal activity and one must be protonated. Therefore, we propose that $\mathrm{EH}$ is the catalytically viable form as opposed the $\mathrm{EH}_{2}$ (in which both are protonated) or $\mathrm{E}$ (in which neither are) (Figure 5). The $\mathrm{p} K_{\mathrm{a}}$ values determined are more consistent with a model containing one glutamic acid ( $\mathrm{p} K_{\mathrm{a}} \sim 5.5$ to 6 ) and one lysine ( $K_{\mathrm{a}} \sim 9.75$ to 10.5 ), as opposed to a model with two glutamic acid residues.

In the equation for fitting of the $\mathrm{pH}$-rate profiles (equation 1), $c$ represents the $\mathrm{pH}$ independent value of $k_{\mathrm{cat}} / K_{\mathrm{m}}$ (or $\left.k_{\mathrm{cat}}\right)$, or more precisely, the limiting value when all of the enzyme is in the active protonation state (EH). The denominator provides the fraction of the free enzyme in this protonation state. When considering the $k_{\text {cat }} / K_{\mathrm{m}}$ data, we calculate a $\mathrm{p} K_{\mathrm{a} 1}=5.43\left(\right.$ or $\left.K_{\mathrm{a} 1}=3.7 \times 10^{-6}\right)$ and a $\mathrm{p} K_{\mathrm{a} 2}=9.92\left(\right.$ or $\left.K_{\mathrm{a} 2}=1.2 \times 10^{-10}\right)$, with a maximal $k_{\text {cat }} / K_{\mathrm{m}}$ value (c) of $19,200 \mathrm{M}^{-1} \mathrm{~s}^{-1}$. The fraction of the $\mathrm{EH}$ active form can be calculated, using the denominator of equation 1 :

$$
1+\left(\frac{H}{K_{a 1}}\right)+\left(\frac{K_{a 2}}{H}\right)=1+\left(\frac{10^{-8}}{3.7 \times 10^{-6}}\right)+\left(\frac{1.2 \times 10^{-10}}{10^{-8}}\right)=1.03
$$

Since this is the denominator, and used to divide our maximal $k_{\text {cat }} / K_{\mathrm{m}}$, this number indicates that $97 \%$ of the enzyme $(1 / 1.03=0.97)$ is in the $\mathrm{EH}$ form at $\mathrm{pH} 8$. It follows that $3 \%$ is in the $\mathrm{E}$ or $\mathrm{EH}_{2}$ forms.

There are two possibilities for $\mathrm{EH}$ : a Glu${ }^{-} / \mathrm{LysH}^{+}$zwitterion or a GluH/Lys neutral form (Figure 5). The most common state at $\mathrm{pH} 8$ would be the $\mathrm{Glu}^{-} / \mathrm{LysH}^{+}$zwitterion form, consistent with a $\mathrm{Glu}^{-}$general base residue that must be deprotonated and $\mathrm{LysH}^{+}$general acid residue that must be protonated for activity. The equilibrium constant for the conversion of the zwitterionic form to the neutral form can be calculated as

$$
\frac{K_{a 2}}{K_{a 1}}=\frac{1.2 \times 10^{-10}}{3.7 \times 10^{-6}}=3.2 \times 10^{-5}
$$

indicating that nearly all is indeed in the zwitterionic form. However, we hypothesize that the GluH/Lys neutral form is the active tautomer, in which the Lys general base must be deprotonated and the GluH general acid must be protonated for activity. The theoretical $k_{\text {cat }} t$ $K_{\mathrm{m}}$ when all enzyme is in the neutral form can be calculated using the equation

$$
c=x * \text { fraction of } E H * \text { fraction of } E H \text { in neutral tautomer }
$$


where $x$ is the $k_{\text {cat }} / K_{\mathrm{m}}$ of the neutral tautomer (GluH/Lys) in the active protonation state (EH). Now, solving for $x$,

$$
\begin{aligned}
& x=c * \frac{1}{\text { fraction of } E H} * \frac{1}{\text { fraction of } E H \text { in reactive tautomer }} \\
& x=c * \frac{1}{0.97} * \frac{1}{3.2 \times 10^{-5}}=19200 \quad M^{-1} s^{-1} * 1.03 * 31000=6.1 \times 10^{8} \quad M^{-1} s^{-1}
\end{aligned}
$$

This value is $\sim 100$-fold less than the diffusion limit of $\sim 10^{9}-10^{10} \mathrm{M}^{-1} \mathrm{~s}^{-1}$, which is the largest possible value for this rate constant. These calculations suggest that our mechanism of acid-base catalysis is possible (a value of $x$ greater than $10^{10} \mathrm{M}^{-1} \mathrm{~s}^{-1}$ would indicate the model unlikely), in which the GluH/Lys is the active conformer, the GluH serves as the general acid and the Lys serves as the general base. These calculations are also consistent with the absent or small solvent isotope effect. The viscosogen data suggest that the reaction is diffusion controlled (microviscogens) and that the effects on substrate binding or product release are not due to major protein conformation changes (macroviscogens). Since the catalyzed reaction is fully reversible, the effect of viscosogens may be related to competition in the active site for chorismate (considered the substrate for this biosynthetic pathway) and isochorismate (product). In light of the $\mathrm{pH}$ rate profiles, the diffusion control is also likely a consequence of requiring an encounter of the substrate with the relatively unpopulated $\mathrm{EH}$ neutral catalytic form of the enzyme.

\section{Conclusions}

The isochorismate synthase PchA uses a (deprotonated) lysine general base residue to activate a water molecular for nucleophilic attack of the chorismate ring at the C-2 position. This is concomitant with a (protonated) glutamic acid serving as a general acid to make the C-4 hydroxyl a good leaving group (Figure 5b). The requirement for the catalytic residues in reverse protonation states is supported by $\mathrm{pH}$ rate profiles, solvent kinetic isotope effect analyses, and viscosogen data. These results can be extrapolated to the other MST family members, providing a family-wide mechanism for the isomerization of the chorismate ring.

\section{Acknowledgments}

We are grateful to A. Ray and A. S. Chilton for technical assistance and to R. L. Schowen for insightful discussions.

$\dagger$ This publication was made possible by funds from NIH grant number P20 RR016475 from the INBRE Program of the National Center for Research Resources, and by NIH grants numbered R01 AI77725 and K02 AI093675 from the National Institute for Allergy and Infectious Disease.

\section{Abbreviations and Full Textual Notes}
EntC isochorismate synthase from E. coli
Irp9 salicylate synthase from Yersinia enterocolitica
MbtI salicylate synthase from Mycobacterium tuberculosis
MenF isochorismate synthase from E. coli
MST protein family containing proteins of menaquinone, siderophore or tryptophan biosynthesis
PchA isochorismate synthase from Pseudomonas aeruginosa 
PchB isochorismate-pyruvate lyase from Pseudomonas aeruginosa

\section{References}

1. He Z, Stigers Lavoie KD, Bartlett PA, Toney MD. Conservation of mechanism in three chorismateutilizing enzymes. J. Am. Chem. Soc. 2004; 126:2378-2385. [PubMed: 14982443]

2. Kolappan S, Zwahlen J, Zhou R, Truglio JJ, Tonge PJ, Kisker C. Lysine 190 is the catalytic base in MenF, the menaquinone-specific isochorismate synthase from Escherichia coli: implications for an enzyme family. Biochemistry. 2007; 46:946-953. [PubMed: 17240978]

3. Zwahlen J, Kolappan S, Zhou R, Kisker C, Tonge PJ. Structure and mechanism of MbtI, the salicylate synthase from Mycobacterium tuberculosis. Biochemistry. 2007; 46:954-964. [PubMed: 17240979]

4. Gaille C, Kast P, Haas D. Salicylate biosynthesis in Pseudomonas aeruginosa. Purification and characterization of PchB, a novel bifunctional enzyme displaying isochorismate pyruvate-lyase and chorismate mutase activities. J. Biol. Chem. 2002; 277:21768-21775. [PubMed: 11937513]

5. Gaille C, Reimmann C, Haas D. Isochorismate synthase (PchA), the first and rate-limiting enzyme in salicylate biosynthesis of Pseudomonas aeruginosa. J. Biol. Chem. 2003; 278:16893-16898. [PubMed: 12624097]

6. Parsons JF, Shi KM, Ladner JE. Structure of isochorismate synthase in complex with magnesium. Acta Crystallogr. 2008; D64:607-610.

7. Sridharan S, Howard N, Kerbarh O, Blaszczyk M, Abell C, Blundell TL. Crystal structure of Escherichia coli enterobactin-specific isochorismate synthase (EntC) bound to its reaction product isochorismate: implications for the enzyme xmechanism and differential activity of chorismateutilizing enzymes. J. Mol. Biol. 2010; 397:290-300. [PubMed: 20079748]

8. Kerbarh O, Chirgadze DY, Blundell TL, Abell C. Crystal structures of Yersinia enterocolitica salicylate synthase and its complex with the reaction products salicylate and pyruvate. J. Mol. Biol. 2006; 357:524-534. [PubMed: 16434053]

9. Kerbarh O, Ciulli A, Howard NI, Abell C. Salicylate biosynthesis: overexpression, purification, and characterization of Irp9, a bifunctional salicylate synthase from Yersinia enterocolitica. J. Bacteriol. 2005; 187:5061-5066. [PubMed: 16030197]

10. Pelludat C, Brem D, Heesemann J. Irp9, encoded by the high-pathogenicity island of Yersinia enterocolitica, is able to convert chorismate into salicylate, the precursor of the siderophore yersiniabactin. J. Bacteriol. 2003; 185:5648-5653. [PubMed: 12949119]

11. Harrison AJ, Yu M, Gardenborg T, Middleditch M, Ramsay RJ, Baker EN, Lott JS. The structure of MbtI from Mycobacterium tuberculosis, the first enzyme in the biosynthesis of the siderophore mycobactin, reveals it to be a salicylate synthase. J. Bacteriol. 2006; 188:6081-6091. [PubMed: 16923875]

12. Chi G, Manos-Turvey A, O'Connor PD, Johnston JM, Evans GL, Baker EN, Payne RJ, Lott JS, Bulloch EM. Implications of binding mode and active site flexibility for inhibitor potency against the salicylate synthase from Mycobacterium tuberculosis. Biochemistry. 2012; 51:4868-4879. [PubMed: 22607697]

13. Lamb AL. Pericyclic reactions catalyzed by chorismate-utilizing enzymes. Biochemistry. 2011; 50:7476-7483. [PubMed: 21823653]

14. Ferrer S, Marti S, Moliner V, Tunon I, Bertran J. Understanding the different activities of highly promiscuous MbtI by computational methods. Phys Chem Chem Phys. 2012; 14:3482-3489. [PubMed: 22307014]

15. Ziebart KT, Toney MD. Nucleophile specificity in anthranilate synthase, aminodeoxychorismate synthase, isochorismate synthase, and salicylate synthase. Biochemistry. 2010; 49:2851-2859. [PubMed: 20170126]

16. Zaitseva J, Lu J, Olechoski KL, Lamb AL. Two crystal structures of the isochorismate pyruvate lyase from Pseudomonas aeruginosa. J. Biol. Chem. 2006; 281:33441-33449. [PubMed: 16914555] 
17. Schmidt K, Leistner E. Microbial Production of (+)-trans-Isochorismic Acid. Biotech. \& Bioeng. 1995; 45:285-291.

18. Luo Q, Olucha J, Lamb AL. Structure-function analyses of isochorismate-pyruvate lyase from Pseudomonas aeruginosa suggest differing catalytic mechanisms for the two pericyclic reactions of this bifunctional enzyme. Biochemistry. 2009; 48:5239-5245. [PubMed: 19432488]

19. Rieger CE, Turnbull JL. Small scale biosynthesis and purification of gram quantities of chorismic acid. Preparative biochemistry \& biotechnology. 1996; 26:67-76. [PubMed: 8744423]

20. Sheely ML. Glycerol viscosity tables. Ind. Eng. Chem. 1932; 24:1060-1064.

21. Segel, IH. Enzyme Kinetics: Behavior and Analysis of Rapid Equilibium and Steady State Enzyme Systems. John Wiley \& Sons; New York: 1975.

22. Schowen KB, Schowen RL. Solvent isotope effects of enzyme systems. Methods Enzymol. 1982; 87:551-606. [PubMed: 6294457]

23. Serino L, Reimmann C, Baur H, Beyeler M, Visca P, Haas D. Structural genes for salicylate biosynthesis from chorismate in Pseudomonas aeruginosa. Mol Gen Genet. 1995; 249:217-228. [PubMed: 7500944]

24. Luo, Q. Molecular Biosciences. University of Kansas; Lawrence, KS: 2009. Mechanistic and structural studies of salicylate biosynthesis in Pseudomonas aeruginosa ( $\mathrm{PhD}$ dissertation).

25. Olucha J, Ouellette AN, Luo Q, Lamb AL. pH dependence of catalysis by Pseudomonas aeruginosa isochorismate-pyruvate lyase: Implications for transition state stabilization and the role of lysine 42. Biochemistry. 2011; 50:7198-7207. [PubMed: 21751784]

26. Schowen RL. The use of solvent isotope effects in the pursuit of enzyme mechanisms. J. Labelled Compd Rad. 2007; 50:1052-1062.

27. Cleland WW. Determining the chemical mechanisms of enzyme-catalyzed reactions by kinetic studies. Adv. Enzymol. Relat. Areas Mol. Biol. 1977; 45:273-387. [PubMed: 21524]

28. Cook, PF.; Cleland, WW. Enzyme Kinetics and Mechanism. Garland Science; New York: 2007.

29. Price NE, Cook PF. Kinetic and chemical mechanisms of the sheep liver 6-phosphogluconate dehydrogenase. Arch. Biochem. Biophys. 1996; 336:215-223. [PubMed: 8954568]

30. Zhang L, Chooback L, Cook PF. Lysine 183 is the general base in the 6-phosphogluconate dehydrogenase-catalyzed reaction. Biochemistry. 1999; 38:11231-11238. [PubMed: 10471272]

31. Dahm C, Muller R, Schulte G, Schmidt K, Leistner E. The role of isochorismate hydroxymutase genes ent $\mathrm{C}$ and menF in enterobactin and menaquinone biosynthesis in Escherichia coli. Biochim. Biophys. Acta. 1998; 1425:377-386. [PubMed: 9795253]

32. Liu J, Quinn N, Berchtold GA, Walsh CT. Overexpression, purification, and characterization of isochorismate synthase (EntC), the first enzyme involved in the biosynthesis of enterobactin from chorismate. Biochemistry. 1990; 29:1417-1425. [PubMed: 2139795]

33. Karsten WE, Lai C-J, Cook PF. Inverse solvent isotope effects in the NAD-malic enzyme reaction are the result of viscosity difference between $\mathrm{D}_{2} \mathrm{O}$ and $\mathrm{H}_{2} \mathrm{O}$ : implications for solvent isotope effect studies. J. Am. Chem. Soc. 1995; 117:5914-5918.

34. Adams JA. Kinetic and catalytic mechanisms of protein kinases. Chem Rev. 2001; 101:2271-2290. [PubMed: 11749373]

35. Caldwell SR, Newcomb JR, Schlecht KA, Raushel FM. Limits of diffusion in the hydrolysis of substrates by the phosphotriesterase from Pseudomonas diminuta. Biochemistry. 1991; 30:74387444. [PubMed: 1649628]

36. Mattei P, Kast P, Hilvert D. Bacillus subtilis chorismate mutase is partially diffusion-controlled. Eur. J. Biochem. 1999; 261:25-32. [PubMed: 10103029]

37. Walsh CT, Liu J, Rusnak F, Sakaitani M. Molecular studies on enzymes in chorismate metabolism and the enterobactin biosynthetic pathway. Chem Rev. 1990; 90:1105-1129.

38. Spraggon G, Kim C, Nguyen-Huu X, Yee MC, Yanofsky C, Mills SE. The structures of anthranilate synthase of Serratia marcescens crystallized in the presence of (i) its substrates, chorismate and glutamine, and a product, glutamate, and (ii) its end-product inhibitor, Ltryptophan. Proc. Natl. Acad. Sci. USA. 2001; 98:6021-6026. [PubMed: 11371633]

39. DeLano, W. The PyMOL Molecular Graphics System. DeLano Scientific; San Carlos, CA: 2002. www.pymol.org 


\section{Highlights}

- The general acid-general base catalysts are identified in MST enzymes.

- Catalysis is diffusion controlled in the isochorismate synthase reaction.

- Evidence for reverse protonation states of the catalytic acid/base is presented. 


\section{A. Isochorismate synthase activity}

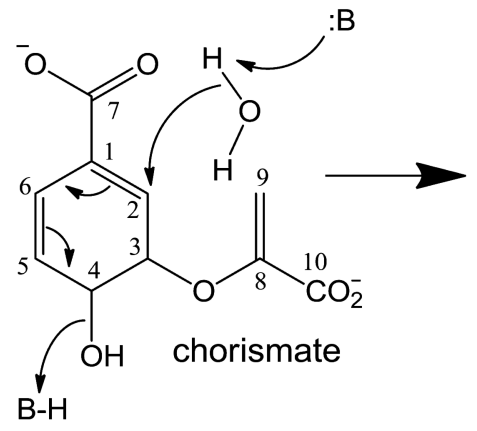<smiles>C=C(OC1C=CC=C(C(=O)[O-])C1O)C(=O)[O-]</smiles>

isochorismate

B. Isochorismate-pyruvate lyase activity

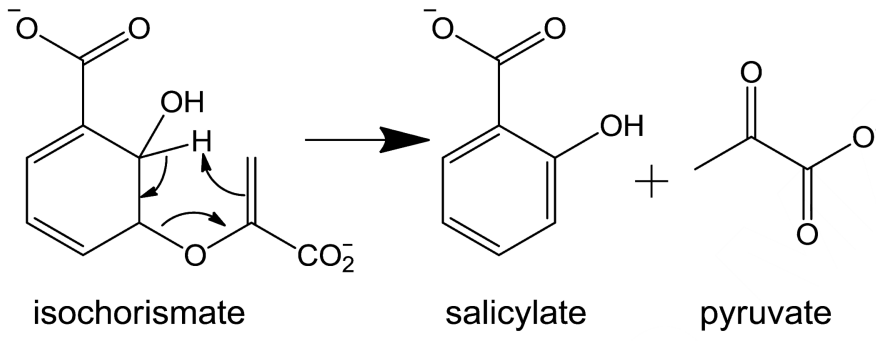

\section{Salicylate synthase activity}

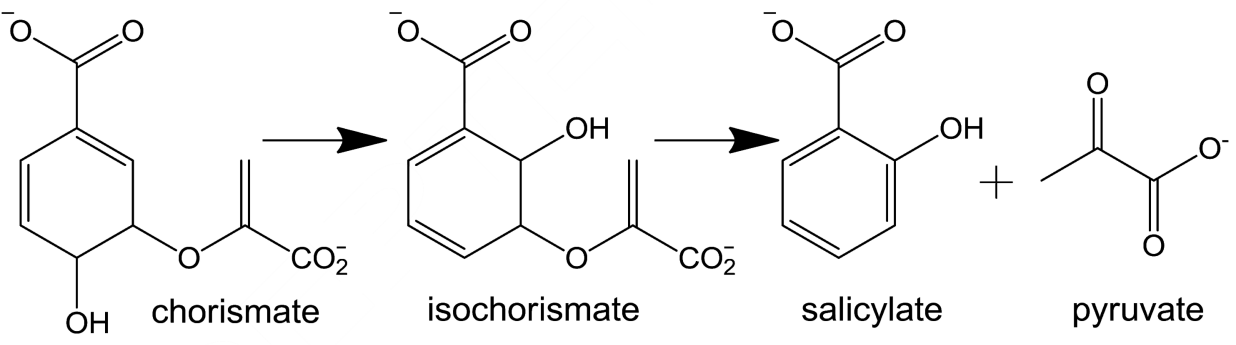

\section{Chorismate mutase activity}

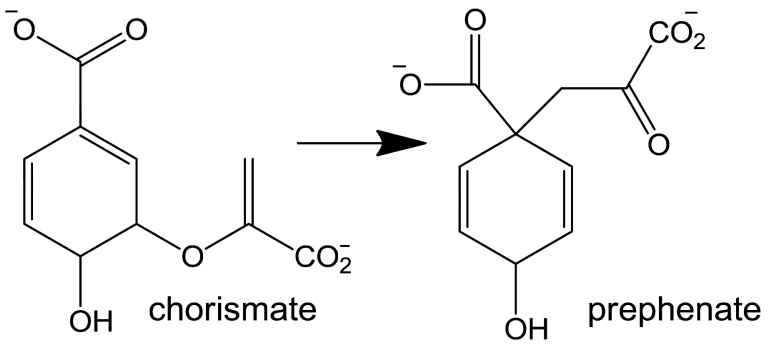

Figure 1.

Reactions catalyzed by isochorismate and salicylate synthases of siderophore and menaquinone biosynthesis. 
A.

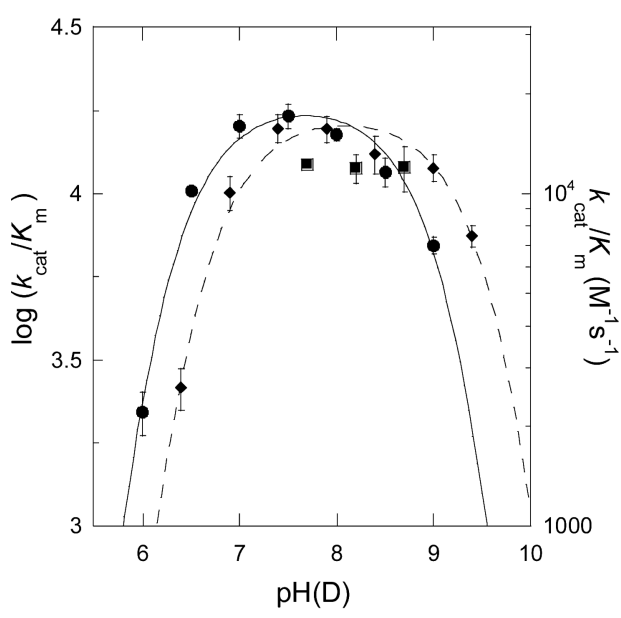

B.

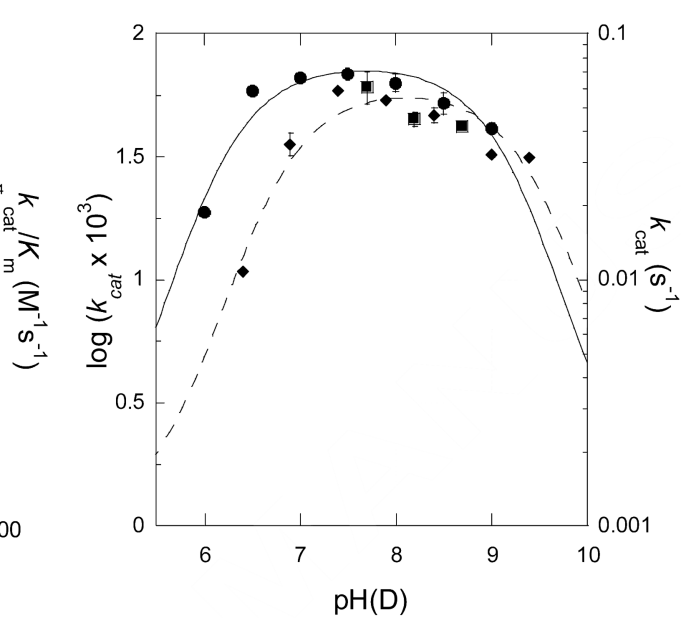

Figure 2. PchA pH and pD rate profiles for $k_{\mathrm{cat}} / K_{\mathrm{m}}(\mathrm{A})$ and $k_{\mathrm{cat}}(\mathrm{B})$ PchA isochorismate synthase activity vs. $\mathrm{pH}$ in $\mathrm{H}_{2} \mathrm{O}(\boldsymbol{O}), \mathrm{D}_{2} \mathrm{O}(\boldsymbol{)})$, and $50 \% \mathrm{D}_{2} \mathrm{O}(\square)$ with curve fit lines for $\mathrm{H}_{2} \mathrm{O}$ (solid line) and $\mathrm{D}_{2} \mathrm{O}$ (dashed lines). The solvent kinetic isotope effect is approximately unity suggesting that the proton transfer steps are not rate limiting. 


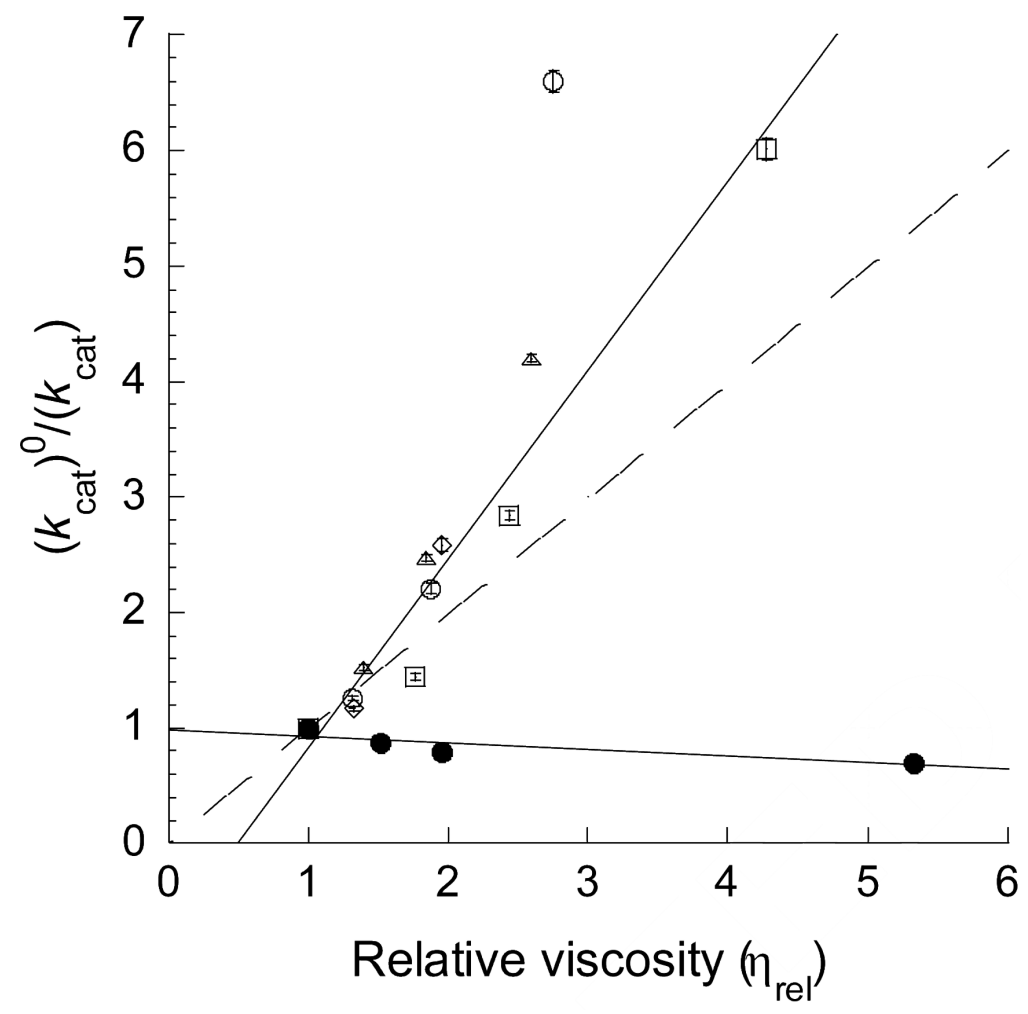

Figure 3.

The effect of viscosogens on the turnover number: glycerol $(\bigcirc)$, sucrose $(\square)$, PEG200 $(\diamond)$, sorbitol $(\triangle$ ), and PEG8000 ( $\mathrm{O}$. The solid lines represent the curve fit for the low-molecular weight viscosogens (open symbols) and the high-molecular weight viscosogen (closed symbols). The dashed line represents the line with a slope of 1 for a completely diffusion controlled rate of reaction. There is a significant effect on the rate of catalysis in the presence of microviscogens in support of the hypothesis that substrate binding or product release is rate limiting. There is no significant effect on the rate of catalysis with macroviscogens, suggesting that a large protein conformational change is not rate limiting. 


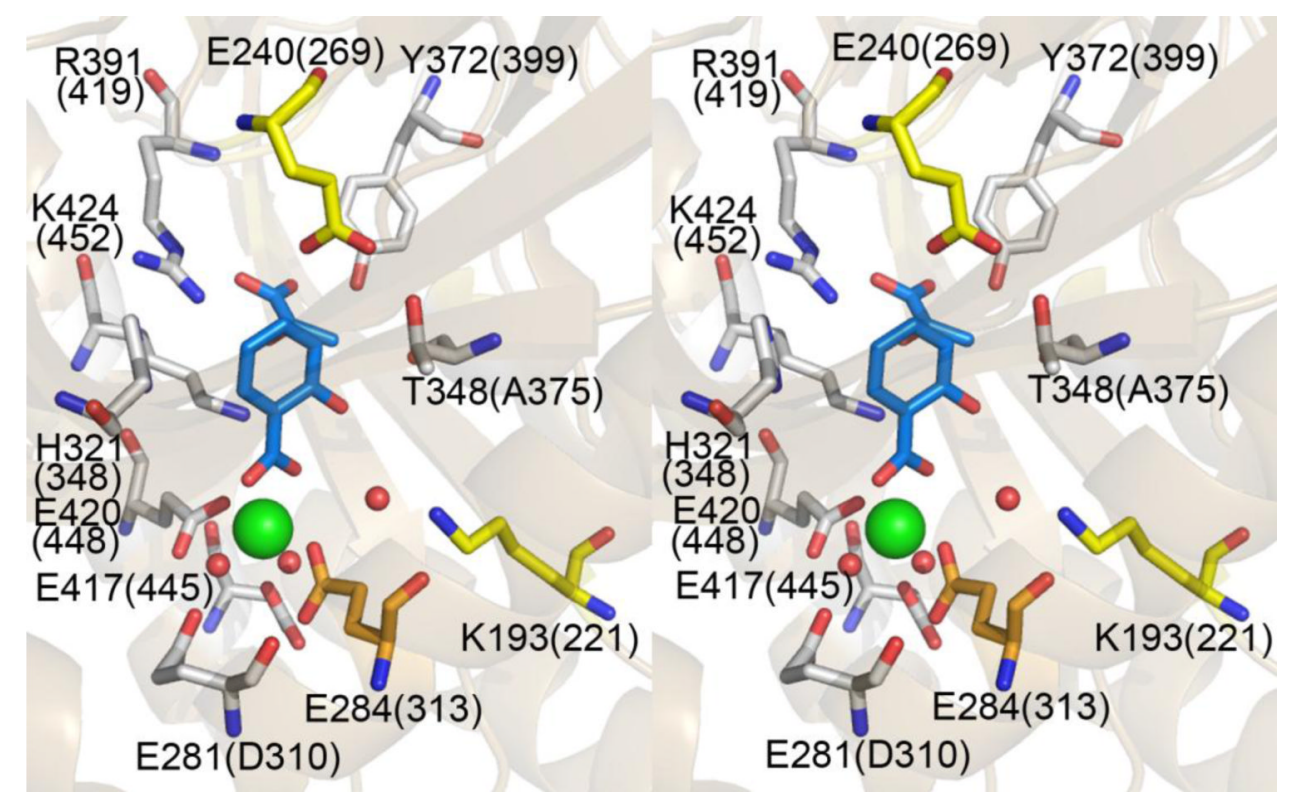

Figure 4. Irp9 active site with proposed general acid-general base residues highlighted The overall structure of Irp9 (PDB: 2FN1) is shown as the transparent cartoon. The active site residues are shown in grey with the exception of the residues of interest herein. Salicylate and pyruvate are blue. The magnesium ions (bright green) and coordinating waters (red) are shown as spheres. Amino acids are labeled with Irp9(PchA) numbering. The residues highlighted in yellow are the proposed general acid, E240(269), and general base, K193(221). Note the water molecule (red sphere) poised between the lysine and salicylate, that has been suggested to be the water activated for catalysis. The general acid proposed from computational experiments that also chelates the metal ion is highlighted in orange, E284(313). This figure was generated with PyMOL ${ }^{(39)}$. 
A.
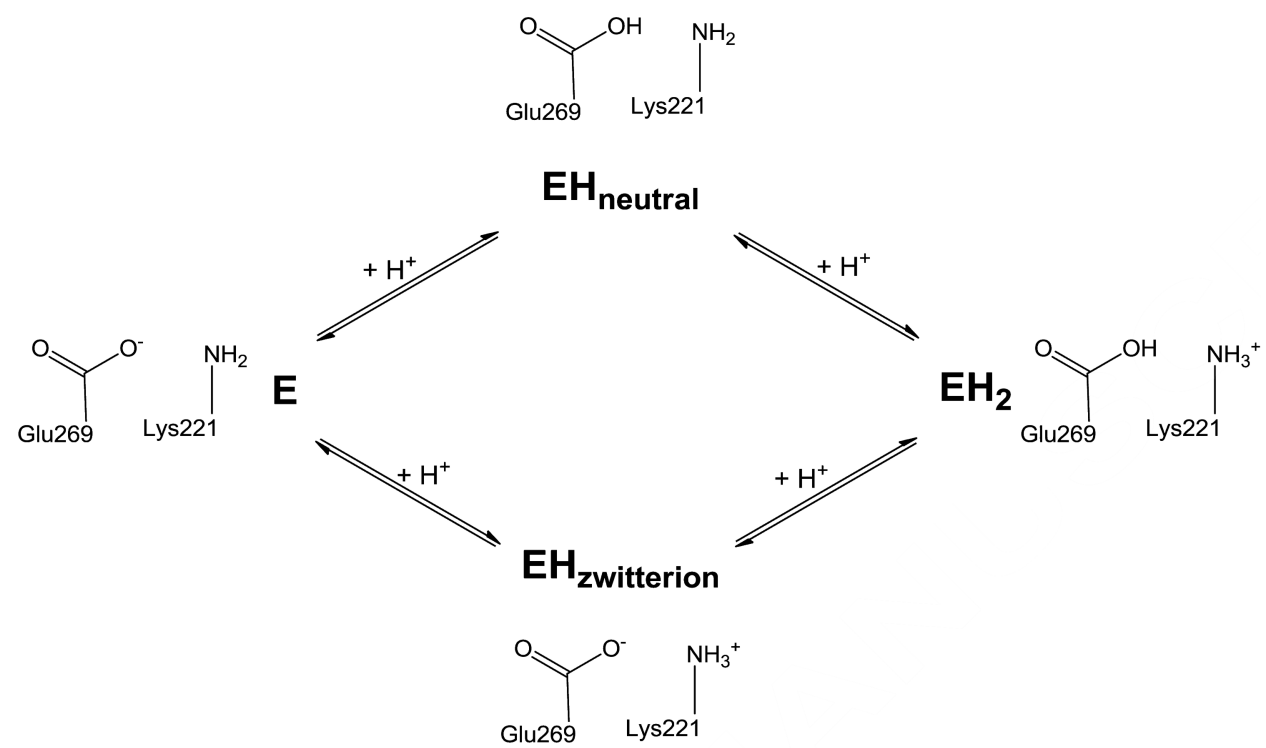

B.

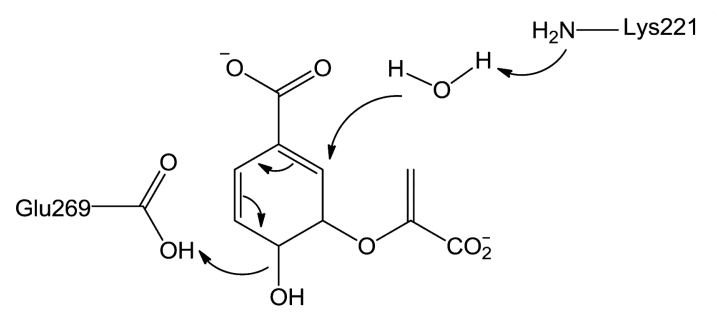

Figure 5.

Proposed Mechanism of Isochorismate Synthase (PchA). 


\section{Table 1}

Primers used to generate wildtype and variant PchA enzymes.

\begin{tabular}{lll}
\hline Amino acid & Putative Function & Primer \\
\hline \multicolumn{2}{l}{$\begin{array}{l}\text { PchA Wildtype } \\
\text { forward }\end{array}$} & 5'-AAT TAT ATA CAT ATG AGC CGG CTG GCG CCC CTG-3'́ \\
reverse & 5'-CCC AAG CTT TCA GGC GAC GCC GCG CTG-3' \\
PchA Variants & \\
K221A $\quad$ General base & 5'-GGG ACG CTT CGG CGC GGT CGT GCT GGC-3' \\
E269A & General acid & 5'-GCG CCT CCC CGG CAC GCC TGG TCC G-3' \\
E313A & Chelates Mg ${ }^{2+}$, general base & 5'-CAA GGA CAG GCA CGC ACA CCA GTT GGT GG-3' \\
E313Q & Chelates $\mathrm{Mg}^{2+}$, general base & 5'-CCA AGG ACA GGC ACC AAC ACC AGT TGG TG-3' \\
\hline
\end{tabular}




\section{Table 2}

Isochorismate Synthase activity for PchA variants of putative general acid - general base amino acids. The assay is a coupled one with 20-fold excess isochorismate-pyruvate lyase ( $\mathrm{PchB}$ ) to convert isochorismate to salicylate, which can be monitored by fluorescence with excitation at $310 \mathrm{~nm}$ and emission at $430 \mathrm{~nm}$.

\begin{tabular}{|l|c|c|c|}
\hline & $\boldsymbol{k}_{\text {cat }}\left(\times \mathbf{1 0}^{\mathbf{- 3}} \mathbf{s}^{\mathbf{- 1}}\right)$ & $\boldsymbol{K}_{\mathbf{m}}(\boldsymbol{\mu M})$ & $\boldsymbol{k}_{\text {cat }} / \boldsymbol{K}_{\mathbf{m}}\left(\mathbf{M}^{\mathbf{- 1}} \mathbf{s}^{\mathbf{- 1}}\right)$ \\
\hline PchA & & & \\
\hline WT & $50.4 \pm 0.2$ & $0.9 \pm 0.1$ & $57,000 \pm 7,000$ \\
\hline K221A & $0.0455 \pm 0.0001$ & $21 \pm 3$ & $2.2 \pm 0.3$ \\
\hline E269A & $\mathrm{ND}^{a}$ & $\mathrm{ND}$ & - \\
\hline E313A & $0.108 \pm 0.001$ & $17 \pm 1$ & $6.4 \pm 0.4$ \\
\hline E313Q & $0.75 \pm 0.04$ & $12 \pm 3$ & $60 \pm 10$ \\
\hline
\end{tabular}

${ }^{a}$ not detected 


\section{Table 3}

$\mathrm{pK}$ values and kinetic constants determined from curve fitting of $\mathrm{pH}$ and $\mathrm{pD}$ rate profiles using equations outlined in the methods.

\begin{tabular}{|c|c|c|c|c|c|}
\hline solvent & $\boldsymbol{k}_{\text {cat }} / \boldsymbol{K}_{\mathbf{m}}$ & $\mathbf{p K}$ value $\left(\boldsymbol{k}_{\text {cat }} / \boldsymbol{K}_{\mathbf{m}}\right)$ & $\boldsymbol{k}_{\text {cat }}$ & pK value $\left(\boldsymbol{k}_{\text {cat }}\right)$ & solvent kinetic isotope effect \\
\hline $\mathrm{H}_{2} \mathrm{O}$ & $19,200 \pm 200 \mathrm{M}^{-1} \mathrm{~s}^{-1}$ & $\mathrm{p} K_{\mathrm{a} 1}=5.43 \pm 0.04$ & $0.076 \pm 0.002 \mathrm{~s}^{-1}$ & $\mathrm{p} K_{\mathrm{a} 1}=5.61 \pm 0.03$ & $\left.\mathrm{D}_{(} \boldsymbol{k}_{\text {cat }} / K_{\mathrm{m}}\right)=1.09 \pm 0.06$ \\
\hline & & $\mathrm{p} K_{\mathrm{a} 2}=9.92 \pm 0.02$ & & $\mathrm{p} K_{\mathrm{a} 2}=9.74 \pm 0.04$ & \\
\hline $\mathrm{D}_{2} \mathrm{O}$ & $17,600 \pm 900 \mathrm{M}^{-1} \mathrm{~s}^{-1}$ & $\mathrm{p} K_{\mathrm{a} 1}=5.77 \pm 0.04$ & $0.0606 \pm 0.0003 \mathrm{~s}^{-1}$ & $\mathrm{p} K_{\mathrm{a} 1}=6.20 \pm 0.03$ & $\mathrm{D}_{(}\left(k_{\text {cat }}\right)=1.25 \pm 0.03$ \\
\hline & & $\mathrm{p} K_{\mathrm{a} 2}=10.41 \pm 0.05$ & & $\mathrm{p} K_{\mathrm{a} 2}=10.01 \pm 0.01$ & \\
\hline
\end{tabular}

